\title{
28 Research Square \\ Oral cenesthopathy proceeding dementia with Lewy body: a case report
}

\author{
Yuta Sawada \\ Sangyo lka Daigaku \\ Yuki Konishi \\ Sangyo Ika Daigaku \\ Atsuko Ikenouchi \\ Sangyo lka Daigaku \\ Reiji Yoshimura ( $\nabla$ yoshi621@med.uoeh-u.ac.jp ) \\ UOEH
}

Case report

Keywords: DLB, DAT, symptom

Posted Date: July 24th, 2020

DOl: https://doi.org/10.21203/rs.3.rs-44107/v1

License: (c) (i) This work is licensed under a Creative Commons Attribution 4.0 International License.

Read Full License

Version of Record: A version of this preprint was published at SN Comprehensive Clinical Medicine on February 16th, 2021. See the published version at https://doi.org/10.1007/s42399-021-00817-3. 


\section{Abstract \\ Background}

We herein report a case of oral cenesthopathy that proceeded dementia with Lewy body (DLB).

\section{Case presentation:}

A 70-year-old female presented with oral cenesthopathy. She was diagnosed with major depression/lateonset schizophrenia and treated with paroxetine and perospirone. Subsequently, she developed sever parkinsonism. Her diagnosis changed to DLB based on clinical features and her magnetic resonance imaging, MIBG scintigraphy, and DAT scans. After tapering off paroxetine and perospirone, the patient was treated with donepezil and levodopa. The DLB symptoms, including oral cenesthopathy and parkinsonism, were relieved.

\section{Conclusion}

This case indicates that oral cenesthopathy may occur as an early symptom of DLB.

\section{Introduction}

Somatic hallucination is defined as the false sensation of an occurrence in the body. When the hallucination is grotesque and visceral, it is classified as a cenesthopathy (1). Cenesthopathy is likely influenced by culture; one study reported that, of the seven unique countries from which patients were recruited, those from Ghana and those with chronic schizophrenia were significantly more likely to report cenesthesia (2).

Dementia with Lewy body (DLB) is a common cause of dementia in the elderly, accounting for $15-25 \%$ of dementia cases (3). Evidence suggests DLB may be underdiagnosed, often being mistaken for mood disorders (4). Some patients are even misdiagnosed with mood disorders or late-onset schizophrenia and treated with antidepressants or antipsychotics (4), which may subsequently worsen parkinsonism. A clinical distinction is important, as it has profound implications for management and prognosis.

We present herein a case of persistent cenesthopathy proceeding DLB that was successfully treated with donepezil and levodopa.

\section{Case Presentation}

A 70-year-old female retired high school teacher presented with complaints of a sensation of her teeth melting and swelling in her gums. At 68 years of age, she consulted a dentist due to the feeling of incongruity in her oral cavity. Although her oral discomfort transiently improved after the oral care 
instructed by the dentist, the symptoms soon relapsed and persisted. She was diagnosed with major depression at an outpatient's clinic, treated with paroxetine ( $20 \mathrm{mg} /$ day), and referred to our university hospital. Her psychiatric symptoms included oral cenesthopathy, depressed mood, persecutory delusion, auditory hallucination, and impaired attention and concentration. Her diagnosis was changed to lateonset schizophrenia, and she was treated with aripiprazole $(24 \mathrm{mg} /$ day) and paroxetine ( $20 \mathrm{mg} /$ day). Her psychiatric symptoms were relieved. Six months later, she again presented with oral cenesthopathy accompanied by persecutory delusion, depressed mood, attention deficits, and anxiety. Aripiprazole was tapered off, and perospirone was gradually increased to $36 \mathrm{mg} /$ day. She then presented with parkinsonism, including finger tremor, muscle rigidity at the upper and lower limbs, bradykinesia, hypersalivation, postural instability, orthostatic hypotension, constipation, and polyuria. Subsequently, she demonstrated rapid eye movement sleep behavior disorder. We reconsidered her diagnosis, and suspected DLB. She retained independence for basic activities of daily living and had no trouble remembering remote past life events; memory problems were restricted to recent events. Her Mini-Mental State Examination score was 27. Magnetic resonance imaging (MRI), single photon emission computed tomography (SPECT), MIBG scintigraphy, and DAT scans were performed. The atrophy of medial central lobe was not pronounced in MRI (Fig. 1). MIBG scintigraphy showed a hypo-accumulation pattern (Fig. 2). Reduced uptake at the lateral basal ganglia was revealed in the DAT scan (Fig. 3). These findings supported the diagnosis of DLB. Perospirone and paroxetine were tapered off, and donepezil was started and increased to $5 \mathrm{mg} /$ day. Four weeks later, the oral cenesthopathy, persecutory delusion, depressed mood, and cognitive flexibility were reduced; parkinsonism, however, persisted. Levodopa (75 mg/day) was added to the ongoing donepezil ( $5 \mathrm{mg} /$ day), and her parkinsonism improved. Written informed consent was obtained from the patient for publication of this case report.

\section{Discussion}

This case sheds light on the rare psychotic symptoms that may occur before the development of DLB. Instead of memory deficits, it is common for the initial cognitive symptoms of DLB to be attention, executive, and visuospatial deficits (5). Psychiatric features are also present in DLB; visual illusion or hallucination, persecutory delusions, depressed mood, and mood fluctuation are often observed (6). Cenesthopathy presents with other psychiatric diseases, including schizophrenia, major depression, and somatoform disorders (7). A recent case report detailed a case of Ekbom syndrome, which consists of the delusion of parasites accompanied by tactile hallucinations, associated with DLB (8). The authors reported rivastigmine, an acetylcholinesterase inhibitor, was effective, and no antipsychotic drugs were needed. Another case of delusional parasitosis in DLB was successfully treated with donepezil and aripiprazole (9). Umezaki et al. (10) first reported a case of oral cenesthopathy that developed into DLB, which was treated with rivastigmine. To the best of our knowledge, the case described herein was the second demonstrating oral cenesthopathy preceding DLB. Although the causal relationship between oral cenesthopathy and DLB remains unclear, close attention should be paid to late-onset psychotic symptoms as a DLB prodrome, even when cognitive impairment is not significant. 


\section{Conclusion}

Our case report indicates that oral cenesthopathy can occur as an early symptom of DLB.

\section{Declarations}

\section{Ethics approval and consent to participate}

Written informed consent was obtained from the patient for publication of this case report.

* Consent for publication

Consent for publication was obtained from all authors.

* Availability of data and material

* Competing interests

The authors have no conflicts of interest to declare.

* Funding

This case report was performed without funding.

\section{* Authors' contributions}

YS was involved in designing the study, collecting the data, and writing the paper. YK and Al assisted with writing the paper. RY was involved in designing the study, supervising data collection, and writing the paper.

\section{*Acknowledgements}

None.

\section{References}

1. Kaplan HI, Sadock BJ. Comprehensive textbook of psychiatry. 5th ed. Baltimore: Williams and Wilkins; 1989.

2. Bauer SM, Schanda H, Karakula H, Olajossy-Hilkesberger L, Rudaleviciene P, Okribelashvili N, Chaudhry HR, Idemudia SE, Gscheider S, Ritter K, Stompe T. Culture and the prevalence of hallucinations in schizophrenia. Compr Psychiatry. 2011;52(3):319-25.

3. Vann Jones SA, O'Brien JT. The prevalence and incidence of dementia with Lewy bodies: a systematic review of population and clinical studies. Psychol Med. 2014;44:673-83. 
4. Walker Z, Possin KL, Boeve BF, Aarsland D. Lewy body dementias. Lancet. 2015;386(10004):168397.

5. Mueller C, Ballard C, Corbett A, Aarsland D. The prognosis of dementia with Lewy bodies. Lancet Neurol. 2017;16(5):348.

6. Morenas-Rodríguez E, Sala I, Subirana A, Pascual-Goñi E, Sánchez-Saudinós MB, Alcolea D, Illán-Gala I, Carmona-Iragui M, Ribosa-Nogué R, Camacho V, Blesa R, Fortea J, Lleó A. Clinical subtypes of dementia with Lewy bodies based on the initial clinical presentation. J Alzheimers Dis. 2018;64(2):505-13.

7. Takahashi T, Fuke T, Washizuka S, Hanihara T, Amano N. A review of recent case reports of cenesthopathy in Japan. Psychogeriatrics. 2013;13(3):196-8.

8. Pontes de Mendonça FJ, Teixeira IA, Marinho V. Ekbom syndrome associated with Lewy body dementia: a case report. Dement Neuropsychol. 2020;14(1):83-7.

9. Ochiai S, Sugawara H, Kajio Y, Tanaka H, Ishikawa T, Fukuhara R, Jono T, Hashimoto M. Delusional parasitosis in dementia with Lewy bodies: a case report. Ann Gen Psychiatry. 2019;18:29.

10. Umezaki Y, Asada T, Naito T, Toyofuku A. A case of oral cenesthopathy in which dementia with Lewy bodies developed during treatment. Psychogeriatrics. 2020.

\section{Figures}

Figure 1

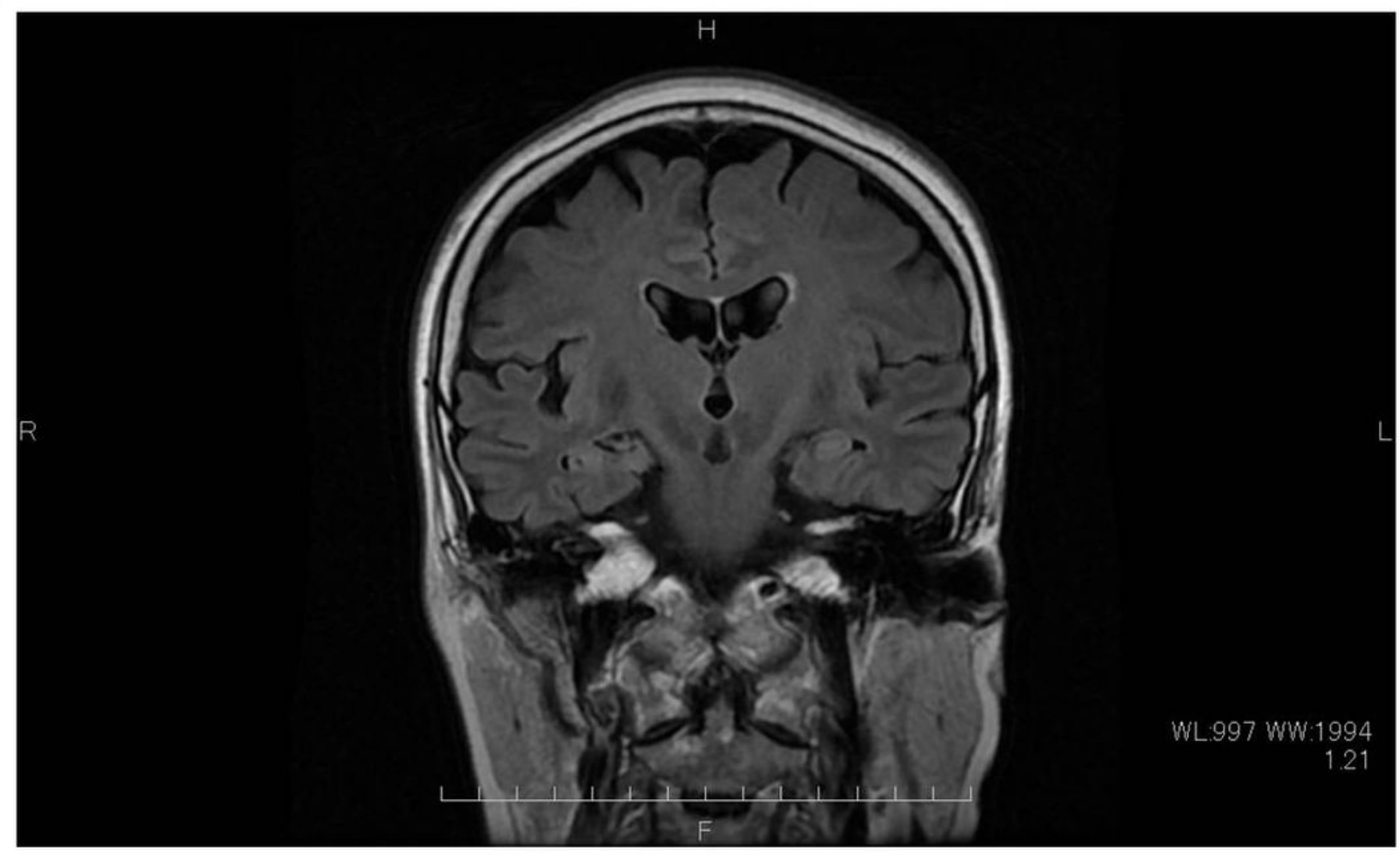

Figure 1 
A magnetic resonance imaging scan shows@@@@@.

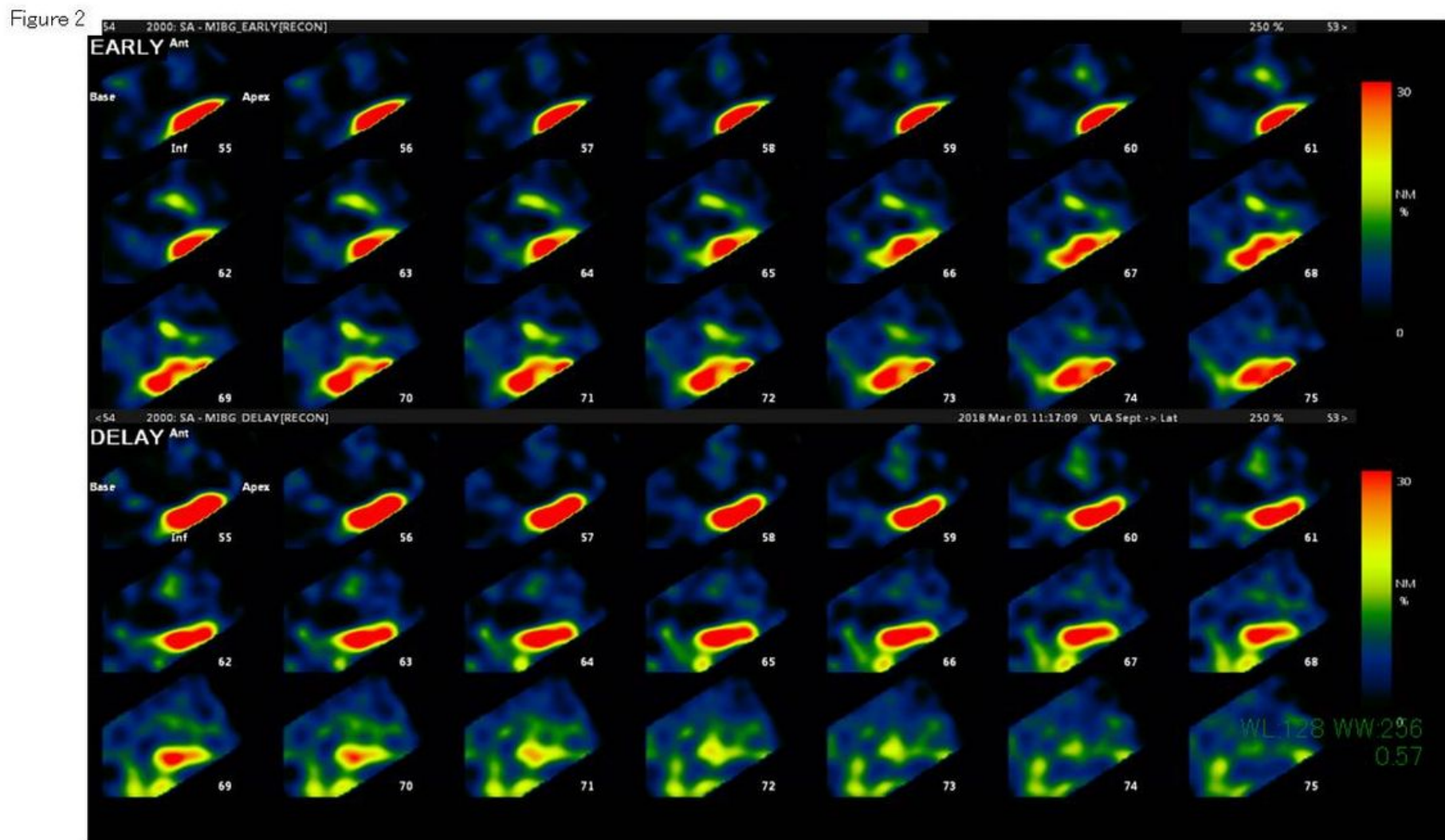

\section{Figure 2}

MIBG scintigraphy shows a significant hypo-accumulation pattern.

Figure 3

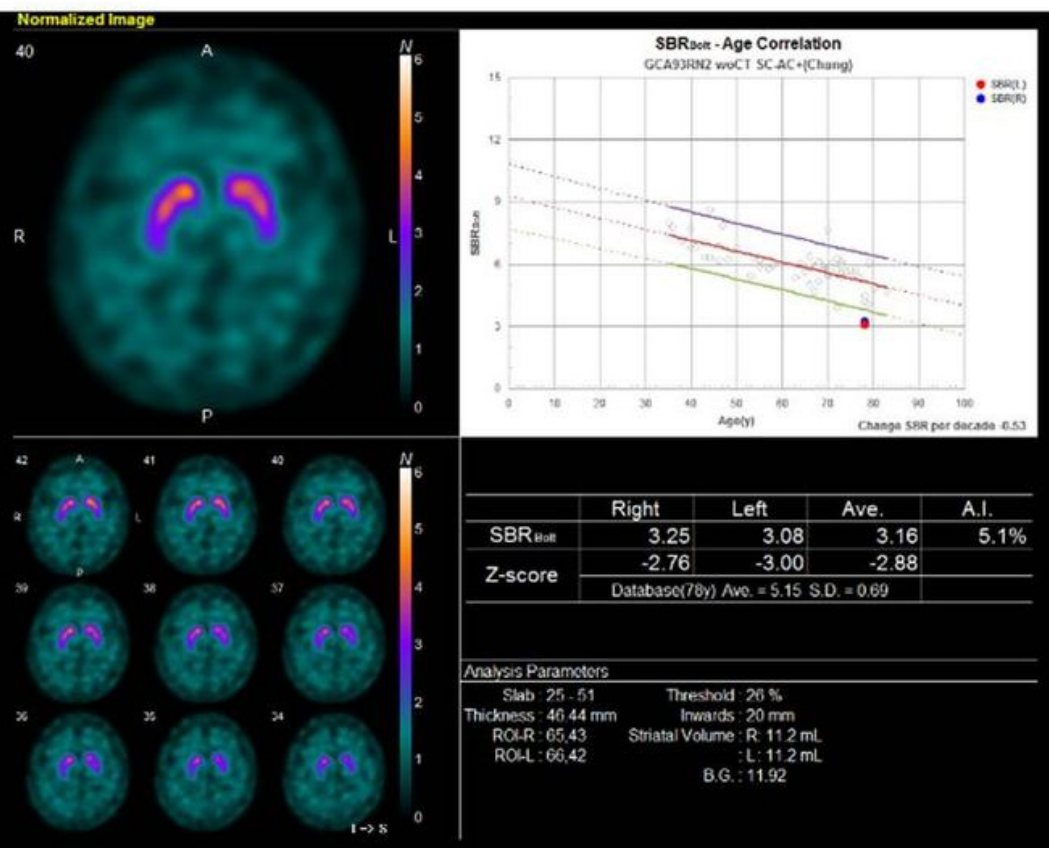


Figure 3

The patient's DAT scan reveals significantly reduced uptake at the bilateral basal ganglia. 